\title{
Anabases
}

ANABASES Traditions et réceptions de l'Antiquité

$25 \mid 2017$

Varia

\section{La République romaine était-elle une république ?*}

\section{Thibaud Lanfranchi}

\section{OpenEdition}

\section{Journals}

Édition électronique

URL : http://journals.openedition.org/anabases/6067

DOI : 10.4000/anabases.6067

ISSN : 2256-9421

\section{Éditeur}

E.R.A.S.M.E.

\section{Édition imprimée}

Date de publication : 1 avril 2017

Pagination : 137-160

ISSN : 1774-4296

\section{Référence électronique}

Thibaud Lanfranchi, "La République romaine était-elle une république ?*», Anabases [En ligne], 25 | 2017, mis en ligne le 01 avril 2020, consulté le 19 janvier 2021. URL : http://journals.openedition.org/ anabases/6067 ; DOI : https://doi.org/10.4000/anabases.6067 


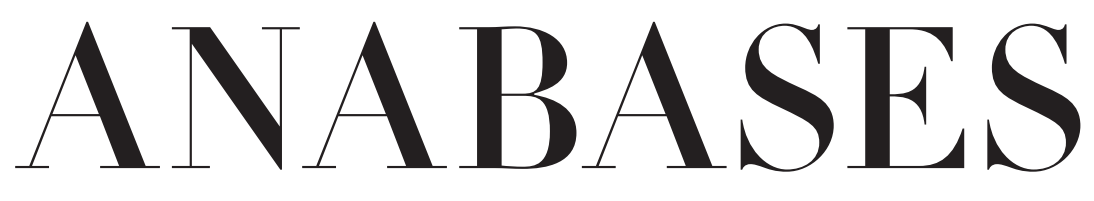

Traditions et Réceptions de l'Antiquité

$$
\begin{aligned}
& N \circ 25 \\
& 2017
\end{aligned}
$$

\section{E.R.A.S.M.E.}

Université Toulouse - Jean Jaurès 



\section{Historiographie et identités culturelles}

Étienne MaIgnan

Un lycéen pas comme les autres à l'école des Anciens :

le « Parallèle littéraire » inédit entre les Iphigénie d’Euripide

et de Racine par Charles Maurras . . . . . . . . . . . . . . . II

Aude Cohen-Skalli

Walter Spoerri (I927-20i6) et les études sur Diodore de Sicile . . . . . . . 59

\section{Traditions du patrimoine antique}

Michel LaCAVE

Anabases en France igoo-20I5. Pour une étude de la réception

de l'Anabase de Xénophon en France : doctrines,

opérations militaires et stratégies . . . . . . . . . . 7I

Loïc Marcou

La réception de l'Antiquité grecque dans le roman policier

néo-hellénique, de Yannis Maris à Petros Markaris . . . . . . . . . . . 95

\section{Archéologie des savoirs}

Romain Millot

Catilina pour combien de temps encore?

Actualités bibliographiques sur un conspirateur trop connu. . . . . . . . III

Thibaud LANFranchi

La République romaine était-elle une république ?. . . . . . . . . . . . I37 


\section{Actualités et débats}

Claude Aziza

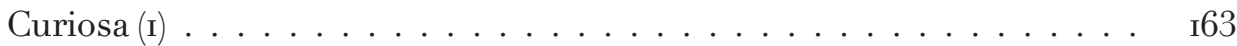

Christine Van Cauwenberghe-HoËT

Marguerite Yourcenar et l'empereur Hadrien,

une réécriture de l'histoire. Exposition au Forum antique de Bavay,

musée archéologique du Département du Nord,

du 4 février au 30 août 20r6. . . . . . . . . . . . . . . . . I 65

Claude AzızA

Antiquités parallèles (6) - Un Jésus insolite. . . . . . . . . . . . . I7 I

\section{Relire les classiques des sciences de l'Antiquité}

Giorgio FerRI

Perché leggere ancora « Quirinus. Una divinità romana

alla luce della comparazione storica » di Angelo Brelich ? . . . . . . . . .

Angelo Brelich

Quirinus. " Una divinità romana alla luce della comparazione storica »,

Studi e Materiali di Storia delle Religioni» XXXI, ı66o, p. 63-ıı . . . . . .

\section{L'atelier de I'histoire : chantiers historiographiques}

L'Antiquité au musée (coordonné par Adeline Grand-Clément) (8)

Pascal CAPus

Chiragan : une nouvelle présentation

des œuvres au Musée Saint-Raymond.

Antiquité et fictions contemporaines (coordonné par Oliviers Devillers) (12)

Silvia STUCCHI

L'image de Rome et de l'empire dans les romans

de Danila Comastri Montanari . . . . . . . . . . . . . . . . . . . . 258

\section{Comptes rendus}

Malika Bastin-Hammou et Charalampos Orfanos (dir.),

Carnaval et comédie (A. Ballabriga) . . . . . . . . . . . 273

Sophie BAsch (éd.), Portraits de Victor Bérard :

actes du colloque international organisé

à l'École française d'Athènes (5-6 avril 2013) (A. Fenet) . . . . . . . . . . . 275 
David J. Breeze, The Roman Army (B. Rossignol) . . . . . . . . . . . . . . . . 277

Luciano CANFora, Il presente come storia. Perché il passato ci chiarische le idee (M. Suzuki) . . . . . . . . . . . . . 278

Juan Ramón carbo Garcia, Apropiaciones de la Antigüedad. De getas, godos, Reyes Católicos, yugos yflechas (G. Reimond) . . . . . . . 28I

Franz Cumont, Astrologie (A. C. D. Panaino) . . . . . . . . . . . . . . . . . 282

Koen De Temmerman et Kristoffel Demoen (éd.), Writing biography in Greece and Rome: narrative technique andfictionalization (M. Cambron-Goulet) .. . . . . . . . . . . . . . . . . . 284

Hans-Christian GüNTHER (dir.), Augustus und Rom : 2000 Jahre Danach (C. Landrea) . . . . . . . . . . . . 286

Steven Hunt, Starting to Teach Latin (Cl. Aziza) . . . . . . . . . . . . . . . . . 287

Ana Iriarte y Luísa De Nazare Ferreiera (coords.), Idades e género na literatura e na arte da Grécia antiga (B. Méndez Santiago) . . . . . . . 288

M. Jankowiak et F. Montinaro, Studies in Theophanes (O. Gengler) . . . . . . 290

Barbara Levick, Catiline (C. Landrea) . . . . . . . . . . . . . . . . . . . . 292

Bruce Lincoln, Politique du paradis. Religion et empire en Perse achéménide (C. Bonnet) . . . . . . . . . . . . . . . . . . . . 294

C. Pisano, Hermes, lo scettro, l'ariete. Configurazioni mitiche della regalità nella Grecia antica (D. Bonanno) . . . . . . . . . . . . . . . 295

Procope de Césaree, Histoire des Goths (O. Gengler) . . . . . . . . . . . . . . 297

Stéphane Ratti, $L$ 'Histoire Auguste. Les païens et les chrétiens dans l'Antiquité tardive (R. Loriol) . . . . . . . . 298

Federico Santangelo, Marius (C. Landrea) . . . . . . . . . . . . . . 3oi

Marisa Tortorelli Ghidini (a cura di), Aurum. Funzioni e simbologie dell'oro nelle culture del Mediterraneo antico (C. Bonnet) . . . . . . . . . . 302

Thijs Weststeisn, Art and Antiquity in the Netherlands and Britain. The Vernacular Arcadia of Franciscus Junius (I59I-I677) (P. Butti de Lima) 3o3 Résumés. . . . . . . . . . . . . . . . . . . . . 305 Index ................................ 3II Errata. . . . . . . . . . . . . . . . . . . . 315 



\section{Archéologie des savoirs}





\section{La République romaine était-elle une république ?*}

Thibaud LANFRANCHI

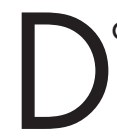
ans ses Prinzipien des römischen Rechts, Fr. Schulz écrit : Die libera res publica der römischen Staatslehre ist mit der “Republik» der modernen Staatslehre nicht identisch; sie läßt mannigfache Ausprägungen zu; und weitgehende Spezialkompetenzen, wie zum Beispiel Sulla erhalten hatte, sind mit ihr verträglich ${ }^{1}$. Cette phrase illustre le problème que je souhaiterais aborder ici : interroger ce que les Romains nommaient la res publica libera, au miroir de ce que nous appelons, en France, “république »². Existe-t-il quelque chose comme une république romaine, non au sens d'un simple système politique, mais au sens plus profond de ce que sous-entend le vocable de république dans la culture politique française? Dans quelle mesure le diagnostic de Fr. Schulz est-il justifié ? La difficulté d'une telle entreprise tient à ce qu'une république - et singulièrement la française - n'est pas seulement un État ou une forme d'État, c'est “ une foi et un idéal, non une réalité ; un contenu politique, social, spirituel même, et non pas seulement une forme $^{3}$ ». Une république est donc à la fois un fait positif, un ensemble constitué d'institutions de type étatique, en même temps que le point de rencontre d'idées

* Ce texte est tiré d'une conférence prononcée le 19 décembre 2013 lors du séminaire “ L’Antiquité, territoire des écarts ». Je tiens à remercier Florence Dupont ainsi que Pierre Vesperini pour m'avoir donné l'occasion de présenter ces réflexions.

1 Fr. Schulz, Prinzipien des römischen Rechts, Munich, 1934, p. 60.

2 Ce choix, volontairement restrictif, se justifie par la volonté de maintenir ce texte dans des limites raisonnables. Prendre en compte toutes les variantes possibles du terme “ république » dans les différents pays du monde pour les comparer à la république romaine nécessiterait un livre en soi.

3 Cl. Nicolet, L'Idée républicaine en France. Essai d'histoire critique, Paris, 1982, p. 161. 
et d'aspirations qui, au fond, peuvent s'avérer assez variées. C'est même, si l'on en croit Gambetta, une forme qui entraîne le fond, c'est-à-dire un régime politique dans lequel architecture institutionnelle et ambitions idéelles sont fortement intriquées. Au-delà des règles institutionnelles (écrites ou non), les pratiques sont donc essentielles à la compréhension de la nature potentiellement républicaine de la République romaine, ainsi qu’à une comparaison avec la république française en particulier. Le sens même de ce qui est appelé “ république » en France est d'ailleurs loin d'être évident, comme en témoignent les multiples substantifs auxquels on accole le terme “ républicain » et qui connaissent, depuis peu, une particulière floraison : pacte républicain, comportement républicain, discipline républicaine, vote républicain, baptême républicain, etc. Comme le remarquait pourtant encore $\mathrm{Cl}$. Nicolet, “ à l'inverse, l'adjectif républicain semble retrouver par instants une valeur précise et unitaire ${ }^{4}$ ». Ajoutons qu'une des originalités du républicanisme français - dès la Révolution - fut de reposer de façon affirmée sur un corps doctrinaire, sur un ensemble de principes, terme auquel il importe de reconnaître un sens presque mathématique synonyme de celui d'axiomes, voire de premiers principes ${ }^{5}$. La Révolution s'accompagna d'un sentiment de fondation nouvelle qui nécessitait de tels principes directeurs, que le républicanisme a recueillis en partage. Plusieurs concepts centraux émergent parmi ces axiomes : la liberté, le rapport au religieux (la fameuse laïcité), l'égalité des droits, l'égalité des chances et la méritocratie, la soumission à la loi, les valeurs sociales ou encore l'attachement à la démocratie. Est-il possible de retrouver de semblables principes à Rome, quand bien même ceuxci n'auraient pas forcément été énoncés ou formalisés comme tels? Est-il possible - et utile - d'essayer de comparer ces deux républiques?

Le voir suppose de revenir sur les grands traits de ce qui nous paraît fonder le régime républicain français pour interroger la situation romaine et, en retour, souligner ce qu'elle peut nous apprendre de nos propres conceptions politiques. Puisqu'une république est à la fois un système politique et une idéologie, ces deux aspects nous retiendront ici, ce qui permettra de contourner le débat sur le caractère plus ou moins démocratique de la République romaine, lequel a produit désormais le meilleur de ses fruits ${ }^{6}$. Cela nous conduira à revenir sur la République romaine en tant que telle, mais par la bande. Ce choix s'explique

\footnotetext{
4 Nicolet, Idée républicaine, p. 10.

5 Nicolet, Idée républicaine, p. 88.

6 On trouvera une utile mise au point sur ce débat chez Fr. Hurlet, “ Démocratie à Rome? Quelle démocratie? En relisant Millar (et Hölkeskamp) », in St. Benoist (éd.), Rome, a City and its Empire in Perspective. The Impact of the Roman World through Fergus Millar's Research, Leyde et Boston, 2012, p. 19-43.
} 
d'abord par le fait que nos connaissances à ce sujet sont à présent bien assurées, même si des points de dissension persistent. Il s'explique aussi parce que l'histoire et le développement de ces deux républiques (française et romaine) furent bien différents. Après un bref retour sur le sens des mots, nous analyserons donc le rapport du régime républicain aux grandes structures qui ont été essentielles dans la construction du républicanisme moderne (l'école, la religion, l'armée), ainsi que sur certaines des valeurs dites républicaines. Notre parcours s'achèvera alors par un retour sur le fonctionnement plus profond de la république romaine. Ce faisant, nous essaierons de montrer en quoi, au-delà de leurs éventuels points communs et divergences, les républiques française et romaine partagent peutêtre un objectif politique d'importance : la neutralisation du politique.

\section{Le sens des mots}

$\mathrm{Au} \mathrm{xx}^{\mathrm{e}}$ siècle, Littré note que le sens premier de "république » dérive du latin res publica, qui signifie chose publique, et désigne donc " toute espèce de gouvernement ", toute forme d'organisation politique de la société. Ce n'est que par un sens particulier que le mot désigne, toujours d'après Littré, le « gouvernement de plusieurs », l'“état gouverné par plusieurs ». Dans un autre sens, dérivé, il désigne toute forme de société humaine, de regroupement de personnes ayant quelque chose en commun (TLF), sens que l'on retrouve dans des formules comme celles de “ république des lettres » ou de « république chrétienne ». Ce second sens nous intéressera moins ici.

Revenons d'abord sur le mot latin, très concret. Sur ce sujet, Cicéron est sans aucun doute le meilleur guide possible. Chez lui, la res publica est la chose publique, c'est-à-dire ce que les gens qui forment communauté ont mis en commun, comme il l'indique dans le De Republica:

Cic., rep., 1, 39 : Est igitur, inquit Africanus, res publica res populi, populus autem non omnis hominum coetus quoquo modo congregatus, sed coetus multitudinis iuris consensu et utilitatis communione sociatus.

Donc reprit l'Africain, la république, c'est la chose du peuple ; mais un peuple n'est pas un rassemblement quelconque de gens réunis n'importe comment; c'est le rassemblement d'une multitude d'individus, qui se sont associés en vertu d'un accord sur le droit et d'une communauté d'intérêts (trad. E. Bréguet) ${ }^{7}$.

Dans un autre passage, Cicéron prolonge cette réflexion en tentant d'établir une distinction entre res publica et ciuitas, mots au sens souvent proche :

7 Voir aussi Cic., off., 1, 17. 
Cic., rep., 1, 41 : Omnis ergo populus, qui est talis coetus multitudinis qualem exposui, omnis ciuitas, quae est constitutio populi, omnis res publica, quae, ut dixi, populi res est, consilio quodam regenda est, ut diuturna sit.

Ainsi tout peuple (c'est-à-dire la multitude qui forme un groupe tel que je l'ai décrit), toute cité (c'est-à-dire ce qui constitue un peuple), toute république (c'est-à-dire, comme je l'ai défini, la chose du peuple) doivent, pour durer, être dirigés selon un certain dessein (trad. E. Bréguet).

E. Lyasse a insisté sur l'aspect concret du terme et sur la continuité qui va de la res priuata à la res publica, la seconde étant, selon lui, « la part de ses biens que le citoyen a en commun avec les autres ${ }^{8}$ ». Comme le note le même E. Lyasse, il est intéressant de constater que c'est le terme de res publica que Cicéron emploie

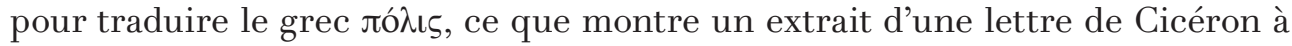
son frère :

Cic., ad Q.fr., 1, 1, 29 : Atque ille quidem princeps ingenii et doctrinae Plato tum denique fore beatas res publicas putauit, si aut docti ac sapientes homines eas regere coepissent aut ii qui regerent omne suum studium in doctrina et sapientia collocassent.

Et à la vérité le grand Platon, ce prince de l'esprit et de la science, estimait que les États ne seraient heureux que quand les savants et les philosophes en auraient pris en main le gouvernement, ou quand leurs gouvernants se seraient donnés tout entiers à la science et à la philosophie (trad. L.A. Constans).

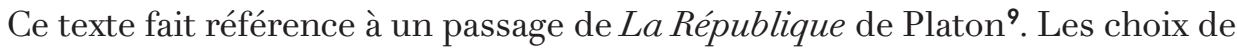
traduction de la notion grecque en latin s'avèrent cependant très variés et ne se

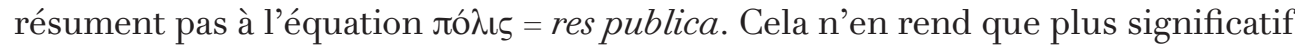
le choix de Cicéron dans ce cas précis ${ }^{10}$.

Le terme de res publica n'est d'ailleurs, par la suite, pas spécifiquement réservé à Rome puisque, sous l'Empire, l'épigraphie démontre qu'il était largement employé pour tous les types de communautés : colonie, municipe, cité pérégrine, etc. Nous n'entrerons pas ici dans les éventuelles variations de sens sous l'Empire. Il convient en revanche d'insister sur le fait que l'expression res publica seule ne peut suffire à qualifier le régime que nous nommons république, raison pour

8 E. Lyasse, “ Les rapports entre les notions de res publica et ciuitas dans la conception romaine de la cité et de l'Empire », Latomus, 66/3, 2007, p. 581-582.

9 Plato, $R$. , 5, 473c : “ À moins, repris-je, que les philosophes ne deviennent rois dans les États, ou que ceux qu'on appelle à présent rois et souverains ne deviennent de vrais et sérieux philosophes, et qu'on ne voie réunis dans le même sujet la puissance politique

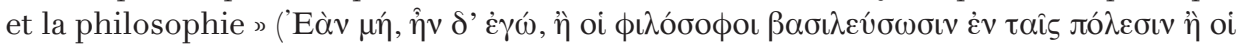

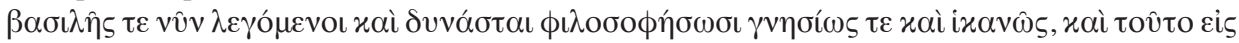

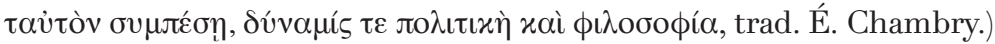

10 Lyasse, “ Les rapports entre les notions de res publica et ciuitas », p. 588-589. 
laquelle nous avons employé plus haut celle de res publica libera. Or une rapide enquête lexicale ne met en évidence que peu d'occurrences de cette expression en latin - trois en tout - toutes tardives. Précisons que, pour cette enquête, n'ont été conservés que les passages où le syntagme res publica libera est explicitement employé, et pas ceux qui pourraient interpréter de la sorte la nature du régime politique romain au moyen de périphrases ${ }^{11}$. Il s'agit des passages suivants :

Cic., Phil., 13, 6 : Sin responderit: «Tu uero ita uitam corpusque seruato, ita fortunas, ita rem familiarem, ut haec libertate posteriora ducas itaque his uti uelis, si libera re publica possis, nec pro his libertatem, sed pro libertate haec proicias tamquam pignora iniuriae », tum sapientiae uocem audire uidear eique uti deo paream.

Mais si elle me répond : “Tu dois en vérité ne conserver ta vie et ton corps, ainsi que tes biens et ton patrimoine, qu'en les faisant passer après la liberté ; ne désire donc en jouir que si tu le peux dans une République libre, et, loin de rejeter pour eux la liberté, rejette-les pour la liberté, comme des gages de l'injustice », alors je croirais entendre la voix de la Sagesse et lui obéirais comme à une divinité (trad. P. Wuilleumier).

Cic., Fam., 11, 3, 4: Nos in hac sententia sumus ut te cupiamus in libera re publica magnum atque honestum esse, uocemus te ad nullas inimicitias, sed tamen pluris nostram libertatem quam tuam amicitiam aestimemus.

Notre façon de voir est la suivante : nous désirons pour toi grandeur et honneur dans une république libre, nous ne t'adressons en aucune façon un appel à l'inimitié ; cependant nous mettons notre liberté au-dessus de ton amitié (trad. J. Beaujeu) ${ }^{\mathbf{2}}$.

Vell., 2, 32 : Digna est memoria Q. Catuli cum auctoritas tum uerecundia. Qui, cum dissuadens legem in contione dixisset esse quidem praeclarum uirum Cn. Pompeium, sed nimium iam liberae rei publicae neque omnia in uno reponenda.

Il convient de rappeler à cette occasion le prestige et la modération de Q. Catulus. Il parlait contre la loi dans une réunion populaire en affirmant que Cn. Pompée était certes une personnalité d'élite, mais déjà trop puissante pour un état libre et qu'il ne fallait pas tout faire reposer sur un seul homme (trad. J. Hellegouarc'h).

Rien avant Cicéron donc et, même dans l'œuvre de Cicéron, l'expression n'apparaît que fort tard. Il faut, bien sûr, se méfier du silence antérieur de nos sources car, avant Cicéron, les auteurs qui auraient pu se pencher sur un tel thème sont rares (Caton par exemple) et leurs textes conservés de façon fragmentaire. Le

1 On trouve en revanche souvent l'expression libera ciuitas qui, pour J. HeLlegouarc'H, Le Vocabulaire latin des relations et des partis politiques sous la République, Paris, 1963, p. 548 désigne aussi le régime romain.

12 Il s'agit cependant d'une lettre publique de M. Junius Brutus et C. Cassius Longinus adressé le 4 août 44 à Marc Antoine. 
contexte d'apparition du terme chez Cicéron - la crise finale du régime républicain - n'en demeure pas moins révélateur. Les Romains de la République semblent donc n'avoir pas eu de mot spécifique pour caractériser leur régime politique, sans doute parce qu'ils n'en éprouvaient pas le besoin. L'expression res publica existait avant Cicéron, mais sans le sens qu'il lui donnait ${ }^{13}$. Cet auteur amorce donc un basculement dans l'usage de cette notion ${ }^{14}$, parce que la période durant laquelle il vécut pouvait pousser à s'emparer de ce terme pour le modifier. C'est en effet seulement une fois ce régime mis en danger qu'il pouvait devenir pensable, ou souhaitable, de mieux le caractériser. Le choix du qualificatif alors accolé à res publica est à ce titre éloquent : libera. C'est l'idée de liberté qui est choisie pour caractériser ce régime. Plusieurs textes le prouvent, à l'instar du début du livre II de l'Histoire romaine de Tite-Live, où l'auteur explique qu'il va désormais retracer l'histoire d'une Rome libre :

Liv., 2, 1, 1 : Liberi iam hinc populi Romani res pace belloque gestas, annuos magistratus imperiaque legum potentiora quam hominum peragam.

C'est une Rome libre dont je vais maintenant retracer l'histoire politique et militaire, sous des magistrats élus pour un an, et sous des lois dont l'autorité est supérieure à celle des hommes (trad. G. Baillet) ${ }^{15}$.

Le premier consul, Iunius Brutus est d'ailleurs qualifié de uindex libertatis ou de liberator urbis ${ }^{16}$. C'est encore plus limpide chez Cicéron, dans le Pro Rabirio où le régime romain est présenté comme le plus libre de tous ${ }^{17}$. Même si l'expression res publica libera est absente de ce dernier passage, elle le sous-tend de bout en bout. Ce texte est également intéressant pour le rapport topographique intime qu'il esquisse entre la res publica et le site de Rome. Ajoutons qu'une partie de la tradition voyait en Servius Tullius, un roi créateur d'institutions typiquement républicaines, le fondateur de la liberté de Rome en insistant précisément sur l'idée que la libertas était l'élément caractéristique du régime républicain romain ${ }^{18}$.

13 H. Drexler, “ Aufstieg und Niedergang Roms in Wechselwirkung mit dem römischen Staatsdenken », $A N R W, 1 / 2$, p. 799-804.

14 Voir Cl. MoAtтı, “Respublica et droit dans la Rome républicaine », MEFRM, 113/2, 2001, p. 811-837.

15 La date de rédaction de la première décade de Tite-Live n'est pas connue avec précision même si nous savons qu'elle date du début de l'époque augustéenne. Voir TiteLive, Histoire romaine. Livre I, texte ét. et tr. par J. Bayet, Paris, 1947, p. XVIXXII.

16 Liv., 2, 1, 8 et Liv., 1, 56, 8. Il n'est de ce point de vue pas anodin qu'Auguste se soit présenté sur certaines monnaies précisément comme le libertatis p(opuli) R(omani) uindex : cf. RPC, I, 2203 (=RIC, $\mathrm{I}^{2}, 79$ Augustus 476).

17 Cic., Rab. post., 22.

18 Cic., Sest., 123 par exemple. Sur ce roi, cf. l'étude fondamentale de R. Thomsen, King 
Notons cependant que le terme de res publica seul pouvait être utilisé pour désigner le régime républicain romain. E. Lyasse le conteste mais il est pourtant assuré que cette connotation du terme existait, seulement à partir de la fin de la République toutefois, comme le prouvent certains textes ${ }^{19}$. Dénier à l'expression res publica toute signification spécifique servant à désigner la République proprement dite est donc une opinion controuvée. Évidemment, dans le cas des auteurs impériaux, spécifier libera aurait pu signifier que l'Empire n'offrait pas la libertas alors même que ce régime entendait préserver une apparence de libertas, comme le prouve l'aureus d'Auguste, porteur de la légende leges et iura $P$. R. restituit ${ }^{20}$. On peut comprendre de la sorte les variations dans l'usage de l'expression. Il n'en demeure pas moins qu'elle put, dès la fin de la République, commencer à désigner, dans certains cas, le régime républicain classique, parfois en lui accolant l'idée de libertas. Ce constat n'ôte rien au fait que l'expression était à l'origine d'une extension plus vague. En l'analysant, Cl. Moatti relève qu'il s'agit d'abord d'un espace, puis d'un espace dans lequel un ensemble de choses sont mises en commun, un espace doté d'une existence matérielle s'incarnant dans une identité politique et une autonomie administrative ${ }^{21}$. Cl. Moatti insiste, à raison, sur l'importance du moment cicéronien dans la théorisation de la res publica, qui eut lieu très tard, mais de façon originale, par la mise en avant de la notion de societas, ainsi que par l'idée d'une création abstraite et volontaire de cette chose commune $^{22}$. Elle insiste de la sorte, à juste titre, sur le fait que cette chose publique ne se comprend à Rome, que dans l'interaction de ses acteurs.

Si l'on en vient alors à la suite de l'histoire du mot en français, on constate que le vocable issu de la soudure des deux termes latins fit son apparition au $\mathrm{xiI}^{\mathrm{e}}$ siècle, mais demeura rare jusqu'au xvi ${ }^{\mathrm{e}}$ siècle, l'usage le réservant plutôt aux antiquités

Servius Tullius. A Historical Synthesis, Copenhague, 1980 et M. Huмm, Appius Claudius Caecus. La République accomplie, Rome, 2005, p. 345-372.

19 Cic., rep., 5, 2 ; Suet., Iul., 77, 1 ou Tac., ann., 1, 3, 7. Voir Fr. Hurlet et B. Mineo, "Introduction », in Eid. (dir.), Le Principat d'Auguste. Réalités et représentations du pouvoir. Autour de la res publica restitua, Rennes, 2009, p. 922 pour une discussion approfondie de ce problème.

20 Voir sur cette monnaie J. W. Rich et J. H. C. Williams, "Leges et iura P. R. Restituit: a New Aureus of Octavian and the Settlement of 28-27 BC », NC, 1999, p. 169-213 et D. Mantovani, "Leges et iura p(opuli) R(omani) restituit. Principe e diritto in un aureo di Ottaviano ", Athenaeum 96, 2008, p. 5-54. Résumé de la discussion dans Hurlet et Mineo, “Introduction », p. 11-17.

21 MoAtт, “Respublica et droit dans la Rome républicaine », p. 814-817.

22 Montтi, “Respublica et droit dans la Rome républicaine », p. 823-824, et p. 825-834 pour une étude de la définition cicéronienne. Voir aussi Cic., off., 1 pour la différence avec communitas. 
romaines. Les définitions plus politiques qui apparurent sont cependant intéressantes. J. Bodin ouvre ainsi son traité des Six livres de la République (1576) : "Republique est un droit gouvernement de plusieurs mesnages, et de ce qui leur est commun, avec puissance souveraine ${ }^{23}$. Tous les mots sont ici pesés au trébuchet: “gouvernement droit» renvoie à un régime réglé par des lois ; "plusieurs mesnages » désigne une société d'hommes assemblés autour de ce qui leur est commun; " puissance souveraine » renvoie au fait que la propriété éminente de la souveraineté revient à cette société d'hommes, qui peut décider de la déléguer. Dans le même ordre d'idée, le dictionnaire de l'Académie (édition de 1694) présente la république comme un " état gouverné par plusieurs », ajoutant que le mot s'emploie parfois "pour toute sorte d'état, de gouvernement» et signalant dès ce moment l'acception secondaire du mot avec l'exemple de la “ république des lettres ». Chez Furetière (édition de 1727), on lit : “ ville ou état libre gouverné par plusieurs, par ses propres citoyens, où l'autorité réside dans le corps du peuple ». Il ressort de ces définitions que, en français, le terme de république désigne à l'origine potentiellement tout type de régime politique (ce qui était déjà le cas de la res publica romaine), du moment qu'il est régi par des lois et que la puissance souveraine y repose, en dernier ressort, sur le peuple. Le terme prit toutefois assez vite un sens plus restreint, désignant les gouvernements par le peuple. C'est la Révolution française qui, en français au moins, consacra définitivement ce sens restreint, conduisant à l'effacement du sens plus large originel contenu dans le latin res publica (chose publique). Stricto sensu, ces définitions n'excluent donc pas forcément les régimes aristocratiques: seules leurs variantes corrompues, telle l'oligarchie, ne peuvent prétendre à l'appellation

23 J. Bodin, Les Six Livres de la République. De Re publica libri sex. Livre premier Liber I, éd. M. Turchetti, Paris, 2013, p. 156. La traduction latine est intéressante: respublica est familiarum rerumque inter ipsas communium summa potestae ac ratione moderata multitudo. En effet, Bodin choisit d'y rendre “ droit gouvernement » par l'idée de moderata ratio qui rappelle directement Cic., rep., 2, 69 : isque concentus ex dissimillimarum uocum moderatione concors tamen efficitur et congruens, sic ex summis et infimis et mediis interiectis ordinibus ut sonis moderata ratione ciuitas consensu dissimillimorum concinit; et quae harmonia a musicis dicitur in cantu, ea est in ciuitate concordia, artissimum atque optimum omni in re publica uinculum incolumitatis, eaque sine iustitia nullo pacto esse potest (“ et ce concert est rendu harmonieux et bien accordé grâce à l'union exactement réglée des voix les plus différentes. Il en est de même dans la cité qui fait naître un accord moral d'éléments très différents, grâce à l'équilibre qui se produit entre les ordres supérieurs, inférieurs et moyens, comme entre des sons divers. Ce que, dans un chant, les musiciens appellent l'harmonie, c'est, dans la cité, la concorde, qui est, pour la sauvegarde de tous, le lien le plus étroit et le plus bienfaisant. Cette concorde n'est réalisable d'aucune manière sans la justice », trad. E. Breguet). 
de république. On le voit de façon éclatante chez Rousseau qui stipule que la monarchie peut être dite république du moment qu'elle est régie par des lois ${ }^{\mathbf{2 4}}$. Rousseau pense cependant essentiellement à des monarchies électives, et non aux monarchies héréditaires.

Liberté, droit, voilà les maîtres mots de la république, l'égalité ne venant qu'après. De fait, la monarchie constitutionnelle établie en France entre 1789 et 1792 présente de larges traits d'une république. Le principe de toute souveraineté est placé dans la Nation, la loi y est comprise comme l'expression de la volonté générale à la formation de laquelle “tous les citoyens ont droit de concourir personnellement ${ }^{25}$ ». Que “ république» puisse désigner à l'origine bien des régimes explique encore le fait qu'après la Révolution, en 1819, Destutt de Tracy puisse proposer une typologie des régimes politiques qui s'écarte délibérément des termes de la trichotomie antique pour diviser les régimes politiques en nationaux et spéciaux, les nationaux étant ceux où le pouvoir appartient au corps entier de la nation, tandis que les régimes spéciaux sont ceux qui lui reconnaissent d'autres sources légitimes (par exemple l'autorité divine ou bien la naissance) ${ }^{26}$. La république entre normalement dans la catégorie des régimes nationaux. Chez H. Kelsen, encore, la république se trouve définie en des termes analogues, comme “ une aristocratie ou une démocratie, selon que le pouvoir souverain appartient à une minorité ou à une majorité du peuple ${ }^{27}$. Contrairement à Rousseau, Kelsen exclut donc la monarchie des républiques possibles. À ses yeux le critère du nombre d'individus investis du pouvoir souverain reste toutefois un critère superficiel, au regard de “ la manière dont la constitution régit la création de l'ordre juridique ", c'est-à-dire du fonctionnement concret du système. Il rappelle enfin implicitement la distinction très nette entre démocratie et république, le second terme pouvant en fait couvrir une bien plus large variété de régimes politiques que le premier.

Qu'est-ce qui est alors républicain? À l'origine substantif, le mot désignait celui qui vit en république, avant de désigner le partisan d'une république. C'est à partir du xvII $^{\mathrm{e}}$ siècle que le terme a commencé à être employé comme un adjectif pour qualifier une personne favorable à la république, puis ce qui est caractéristique de l'état d'esprit des partisans de la république. C'est à partir de là qu'il a pu commencer à se multiplier et à perdre en précision. Le terme de cette évolution est la situation actuelle, où le terme est appliqué un peu partout.

24 J.-J. Rousseau, Du Contrat Social, II, 6, in Id., Euvres complètes, 3, Paris, 1964, p. 379380.

25 Voir Nicolet, Idée républicaine, p. 399.

26 A. Destutt de Tracy, Commentaire sur L'Esprit des lois de Montesquieu, Paris, 1819, p. $12-15$.

27 H. Kelsen, Théorie générale du droit et de l'État, Paris, 1997, p. 332. 
À l'issue de cette première analyse, la République romaine a toute légitimité à être dite républicaine puisque c'est après tout un régime politique régi par des lois (ainsi que par des coutumes), et qui est fondé sur un principe de souveraineté populaire. Si l'on accepte de ne pas confondre souveraineté populaire et mode de gouvernement démocratique ${ }^{28}$, alors il y a bien là une république. Cette idée est d'ailleurs confirmée par le fait que le principat conserva tous les éléments du triptyque magistrature/Sénat/assemblées du peuple, sauf précisément ces dernières, remplacées d'une certaine façon par le princeps lui-même. L'absence de constitution durant la Rome républicaine ne saurait fournir de contreargument ${ }^{29}$. S'il s'agit là d'une grande différence avec la république française issue de 1958, cette situation est finalement assez proche de la III ${ }^{e}$ République $^{30}$. Bien des régimes politiques actuels n'ont en outre pas de constitution. À Rome, cela va même au-delà : il y a une défiance romaine vis-à-vis de ce type de systématisation.

\section{Aspects du républicanisme}

Ces premiers jalons posés, il est possible d'en venir à des aspects plus fondamentaux de ce que l'on nomme républicanisme. Le républicanisme français est bâti sur un corps de valeurs, dont sa devise fournit un bel exemple : liberté, égalité, fraternité. L'idée de liberté est aussi d'une grande importance à Rome, et pas seulement parce que la revendication de libertas fut l'un des thèmes les plus importants de la propagande politique à la fin de la République, particulièrement chez les populares. Nous avons vu qu'elle put servir à préciser la nature du régime antérieur au principat. Cette libertas romaine n'est en revanche pas celle qui est théorisée par les Grecs. Si la libertas désigne effectivement l'autonomie du citoyen, c'est une autonomie conçue et construite dans l'interdépendance visà-vis de ses concitoyens ${ }^{31}$. C'est une liberté qui n'exclut ni la hiérarchie ni la prédominance de certaines portions de la population (par exemple les sénateurs) sur d'autres. Cette hiérarchie est même partie prenante de cette interdépendance dans laquelle est conçue la libertas romaine. Comme l'écrit Fr. Schulz: Dem

28 Voir J.-L. Ferrary, « L'iter legis, de la rédaction de la rogatio à la publication de la lex rogata », in Id., Leges publicae. La Legge nell'esperienza giuridica romana, Pavie, 2012, p. 24-32.

29 Voir la fameuse demande de Pompée à M. Varro, rapportée par Gell., 14, 7, 2 : le jeune Pompée, devenu consul en 70 avant J.-C., sollicita en effet Varron pour qu'il lui compose un commentarium sur la façon de présider et de consulter le Sénat. Varron s'exécuta et mit donc par écrit les règles non-écrites régissant le fonctionnement de cette institution.

30 Nicolet, Idée républicaine, p. 166-167 où il emploie même le mot de coutume.

31 Hellegouarc' ', Vocabulaire latin, p. 543. 
römischen Freiheitsbegriff ist die Begrenzung immanent ${ }^{32}$, de sorte que la liberté est constitutive de l'appartenance au populus et à la res publica. J. Hellegouarc'h l'indique de façon on ne peut plus claire : “Il n'y a pas de res publica sans libertas ${ }^{33}$ ». Ajoutons que si la libertas est propre à tous les citoyens, elle est par excellence la qualité de la plèbe qui n'a pas la dignitas et pour qui elle représente ce qui la fait appartenir à la res publica. De la sorte, la libertas romaine est un concept moins politique que juridico-pratique (“praktisch-juristisch »), essentiellement opérant, pour reprendre la terminologie de Fr. Schulz, dans le droit constitutionnel romain $^{34}$ : c'est la liberté de prendre part à la formation de la volonté de l'État et de ne pas avoir un dominus au-dessus de soi. Ce n'est pas faire ce que l'on veut lorsqu'on le veut. Il faut ainsi tenir compte des devoirs envers l'État (notamment militaires) et des bornes imposées par toute une série d'éléments comme la fides, la pietas ou l'auctoritas. Le principe de liberté s'intrique avec ces autres principes, ce qui explique pourquoi il s'agit d'un terme opérant à l'intérieur du droit constitutionnel romain.

La fraternité est totalement inconnue des Romains. Ce n'est pas le cas de l'égalité, même si cette égalité est bien particulière : c'est la fameuse égalité géométrique chère à Cl. Nicolet sur laquelle il n'est pas utile de revenir tant elle est bien connue à présent. Rappelons simplement qu'elle s'incarne de la façon particulièrement nette dans le système de vote des comices centuriates : devoirs et responsabilités civiques y sont proportionnés à la hiérarchie du cens et donc à la fortune. Là se révèle la structure censitaire profonde de la République romaine, là se dévoile son caractère de république aristocratique qui ne fut jamais remis en cause $^{35}$.

On pourrait penser ensuite à plusieurs valeurs plus souterraines, dont une, assez centrale de nos jours (au moins pour en déplorer la disparition) : la méritocratie. Cette notion désigne un système qui tend à permettre la promotion des individus dans l'échelle sociale en raison de leurs mérites respectifs. Citons, à ce propos, le titre premier de la constitution de 1791 : « La Constitution garantit, comme droits naturels et civils : $1^{\circ}$ Que tous les citoyens sont admissibles aux places et emplois, sans autre distinction que celle des vertus et des talents ». Dans une société comme celle de Rome, semblable idée pourrait paraître totalement anachronique, même si elle a récemment été creusée dans une thèse de doctorat non encore publiée et consacrée aux procédures de dégradation des citoyens, en

32 Schulz, Prinzipien, p. 95.

33 Hellegouarc' H, Vocabulaire latin, p. 545.

34 Schulz, Prinzipien, p. 96.

35 La présentation de ces problèmes dans Cl. Nicolet, Le Métier de citoyen dans la Rome républicaine, Paris, 1976 demeure un bon point de départ. 
lui accolant justement le qualificatif de géométrique : il y aurait donc eu à Rome, outre l'égalité géométrique bien connue, une forme de méritocratie géométrique ${ }^{36}$. Cette méritocratie serait le fruit progressif d'un plébiscite d'une importance exceptionnelle : le plébiscite ovinien qui confia la rédaction de l'album sénatorial (ce que les Romains appellent la lectio senatus) aux censeurs, sans doute entre 318 et 312 avant J.-C. Ce plébiscite nous est connu uniquement par un texte fragmentaire de Festus, lequel a fait l'objet de vives discussions sans qu'un accord soit intervenu à ce jour :

Fest., p. 290 L., s.v. Praeteriti senatores : praeteriti senatores quondam in opprobrio non erant, quod, ut reges sibi legebant, sublegebantque, quos in consilio publico haberent, ita post exactos eos consules quoque et tribuni militum consulari potestate coniunctissimos sibi quosque patriciorum, et deinde plebeiorum legebant; donec Ouinia tribunicia interuenit, qua sanctum est, ut censores ex omni ordine optimum quemque curiatim in senatum legerent. Quo factum est, ut qui praeteriti essent et loco moti, haberentur ignominiosi.

Il y eut une époque où les sénateurs exclus <du Sénat> n'étaient pas jetés dans l'opprobre, étant donné que les rois recrutaient et remplaçaient par eux-mêmes ceux qui participaient au conseil public, et après l'éviction des rois, les consuls aussi, ainsi que les tribuns militaires à pouvoir consulaire, choisissaient de même chacun de leurs très proches amis parmi les patriciens, puis parmi les plébéiens ; jusqu'à ce que la loi du tribun Ovinius intervînt, par laquelle il était prescrit que les censeurs choisiraient pour faire partie du Sénat chacun des meilleurs de chaque ordre en procédant par curie. À partir du moment où ceci fut mis en application, ceux qui étaient exclus $<$ du Sénat> et évincés de leur rang étaient frappés d'ignominie (trad. M. Humm).

Tous les historiens s'accordent sur la mesure principale imposée par ce plébiscite, à savoir le transfert de la désignation des sénateurs (la lectio senatus), des consuls (ou des détenteurs de l'imperium) aux censeurs. Sans entrer ici dans toutes les difficultés posées par ce texte, soulignons la principale qui soit pertinente pour notre propos : l'interprétation de la formule ex omni ordine. Ce membre de phrase ne peut s'interpréter que de deux façons : soit en lui donnant un sens distributif, soit en en faisant un singulier. Le premier sens, privilégié notamment par T. J. Cornell, est toutefois plus convaincant ${ }^{37}$. Il faut donc comprendre le texte

36 Cl. Bur, La Citoyenneté dégradée: recherches sur l'infamie à Rome de 312 av. à 96 ap. J.-C., thèse dactylographiée de l'Université Paris 1 Panthéon-Sorbonne, 2013. Cette idée du caractère méritocratique de la nobilitas, à savoir de l'importance des accomplissements individuels, avait cependant déjà été proposée par K.J. HöLKEsKamP, Die Entstehung der Nöbilität. Studien zur sozialen und politischen Geschichte der Römischen Republik im 4. Jh. v. Chr., Stuttgart, 1987, p. 241-258.

37 T. J. Connell, "The lex Ovinia and the Emancipation of the Senate ", in Chr. Bruun (éd.), The Roman Middle Republic. Politics, Religion and Historiography, c. 400-133 B.C. 
de Festus de la façon suivante : le plébiscite d'Ovinius imposait aux censeurs de choisir les sénateurs ex omni ordine ciuitatis, ce qui signifie qu'il fallait les recruter parmi tous les citoyens quel que fût leur rang. Que signifie alors la formule optimum quemque? Indique-t-elle une comparaison des citoyens par le censeur ou s'agit-il d'une allusion à une classe considérée comme celle des meilleurs? La première solution prévaut en général, ce qui signifie que la disposition d'Ovinius modifia le mode de désignation des sénateurs et précisa les critères de choix à partir desquels les censeurs devaient opérer : ils devaient désormais recruter les meilleurs, ce qui aboutit à l'apparition du regimen morum des censeurs. Pour ce faire, les mérites individuels étaient pris en compte et avaient de l'influence ${ }^{38}$.

Cette idée de méritocratie géométrique a donc son intérêt pour peu qu’on en comprenne bien le sens. Les ressources pécuniaires, le capital social, demeuraient des éléments déterminants de la place dans la société, chose naturelle dans une société censitaire. Dans un second temps toutefois, par l'introduction de ce recrutement des meilleurs, un élément de méritocratie pouvait jouer dans l'attribution des honneurs. Entre deux aristocrates, on choisissait celui qui avait (ou plutôt était supposé avoir) les vertus les plus éclatantes. Le mérite en question jouait ainsi pour tous, mais de façon différenciée selon les couches sociales et en fonction de la position originelle dans la hiérarchie sociale. Il ne s'agissait donc pas d'une méritocratie complète, contrairement à l'idéal méritocratique français dans lequel le fils d'ouvrier peut, en théorie, devenir président d'une multinationale. Cela était impossible à Rome où la méritocratie était aussi un élément de contrôle social, de reproduction sociale, en ce sens qu'elle servait à justifier la prééminence des sénateurs, puisqu'ils étaient bien les meilleurs. D'où l'importance des procédures d'infamie et leur publicité lorsqu'il s'agissait des premiers éléments de la cités9.

Bien qu'il s'agisse là d'une idée pouvant surprendre, il est possible d'en tester la pertinence à partir de l'exemple de Cicéron. C'est moins son parcours en lui-même que ses origines qui sont révélatrices puisque certaines sources en font le descendant d'un foulon (Plutarque, qui rapporte ici un discours de Q. Fufius Calenus $)^{40}$. Ne nous y trompons pas, le registre du discours de Calenus est celui de la polémique puisqu'il attaque la vénalité supposée de Cicéron. Il est donc obligé de forcer le trait sur ses origines, pour pouvoir accentuer l'image de cette vénalité.

(Papers from a Conference at the Institutum Romanum Finlandiae, September 11-12, 1998), Rome, 2000, p. 69-89.

38 Voir plus largement sur ce plébiscite Bur, Citoyenneté dégradée et Th. Lanfranchi, Les Tribuns de la plèbe et la formation de la République romaine (494-287 avant J.-C.), Rome, 2015, p. 331-337.

39 Bur, Citoyenneté dégradée, p. 48, p. 77 et p. 881.

40 Plut., Cic., 1, 12 et D.C., 46, 4, 2. 
Si cette légende du père foulon, apparue assez tôt, est donc vraie, il faut plutôt imaginer que le père de Cicéron possédait des fouleries : il entrerait de la sorte dans la catégorie que N. Tran qualifie d'entrepreneurs ${ }^{41}$. Il n'en demeure pas moins intéressant que le parcours de Cicéron n'a pas pu se bâtir seulement sur l'argent rapporté par cette activité. Il lui a fallu en gagner (d'où l'accusation de vénalité) et faire la preuve de ses mérites pour se créer des clientèles indispensables à son parcours politique. Cicéron se vantait d'ailleurs d'être le premier homo nouus à avoir obtenu le consulat depuis longtemps ${ }^{42}$. De fait, la préture constituait souvent la charge la plus haute que cette catégorie de personnages pouvait atteindre, sauf à disposer de mérites éclatants. De façon comparable, un personnage au destin plus grand encore eut à subir semblables accusations. Marc Antoine reprochait ainsi à Octave d'avoir eu pour bisaïeul un affranchi, un cordier de Thurium et pour grand-père un changeur ${ }^{43}$. Nous sommes ici, une fois de plus, dans le registre polémique et il faut se méfier d'un trait qui doit être forcé. Antoine ne put toutefois forger cette histoire à partir de rien. Au-delà du concept de méritocratie géométrique, que nous empruntons à Cl. Bur, peut-être nous faudrait-il aussi envisager une dimension générationnelle qui semble émerger de ces exemples : l'accumulation primitive du capital économique permet ensuite de le valoriser dans le champ social, les mérites individuels permettant seuls de franchir les derniers échelons. Cette méritocratie géométrique apparaît de la sorte aussi comme une méritocratie héréditaire, qui incarne le caractère aristocratique de la République romaine, même si sur le fond, elle n'est peut-être guère plus qu'une fiction que l'aristocratie romaine construit et projette, pour justifier sa propre domination.

Reste le rapport à certaines institutions constitutives d'une société où, cette fois, les différences entre Rome et la république française sont manifestes. De ce point de vue, le rapport à l'instruction témoigne d'une différence fondamentale. Dès la Révolution, avec Condorcet ou avec des idéologues comme Daunou, la question de l'instruction et d'un état élevé de l'opinion fut perçue comme nécessaire à un régime de liberté. Ce programme fut constamment repris par les républicains successifs et fut la grande affaire de la III ${ }^{\mathrm{e}}$ République. L'instruction publique est l'un des points de consensus majeur du républicanisme français. L’idée profonde qui la sous-tend est que seule « une éducation de masse, fondée sur une pédagogie scientifique» peut permettre “l'accomplissement irréversible des principes énoncés et annoncés en 1789 », c'est-à-dire d'un authentique républicanisme : “ Il n'y a de République et de démocratie que par l'école ${ }^{44}$. „C'est encore valable

41 Voir aussi E. NArducci, Cicerone. La parola e la politica, Bari, 2009, p. 22-24.

42 Cic., leg. agr., 2, 1, 3.

43 Suet., Aug., 2, 6.

44 Nicolet, Idée républicaine, p. 288. 
aujourd'hui, comme le montre les débats récurrents autour de certaines institutions scolaires comme les classes préparatoires aux grandes écoles. Ainsi, parmi les innombrables " points de vue » sur ce sujet publiés dans les journaux, mentionnons celui de Cédric Allmang (professeur de CPGE au lycée Saint-Louis de Paris) dans lequel on peut lire la phrase suivante: “ Mais ces conditions de travail, ce lien élève professeur, cet idéal républicain qui prône la réussite par le savoir est tellement rare que ceux qui le perpétuent apparaissent comme des privilégiés. » Et d'ajouter plus loin : « Aujourd'hui, ce dernier bastion des hussards noirs de la République est présenté, par le ministre qui en a la charge, comme une aberration, une somme de conservatismes et de prébendes qu'il faut casser ${ }^{45}$. ” Au-delà d'une rhétorique un peu lourde, soulignons la persistance de quelque chose qui n'existe nullement à Rome où la démopédie n'intéresse personne. L'éducation n'est à aucun moment perçue comme un élément nécessaire du système politique et relève des particuliers ${ }^{46}$. Inversement, pour les idéologues, il y a un renversement complet du modèle proposé par la philosophie de Platon : la " République » ne doit pas être dirigée par un philosophe mais constitue en revanche le système politique qui, d'une certaine manière, nécessite que tous les citoyens soient des philosophes et qui confère une importance cruciale à la science $^{47}$. De ce point de vue, le modèle éducatif de nos républicains tient plus de la paideia des cités grecques.

Sur la religion, le républicanisme moderne n'a pas, si l'on peut dire, de religion définie. Le cas français est même ici, au contraire, fort spécifique en ce qu'il veut couper les liens entre l'État et toute religion, établie ou non. La séparation des églises et de l'État est une chose trop connue pour qu'on ait besoin d'y insister ${ }^{48}$. Il est également bien connu qu'il s'agit là d'une singularité que l'on ne retrouve guère ailleurs. L'Allemagne ou les États-Unis d'Amérique offrent des exemples bien différents et tout aussi républicains. Rome s'inscrit de fait également dans une optique radicalement différente. Le fas y est une part du ius et la séparation des deux fut extrêmement tardive ${ }^{49}$. D'après Cicéron, P. Scaevola (le consul de 133) avait coutume de dire que l'on ne pouvait être un bon pontife si on ne connaissait

45 http://www.lemonde.fr/idees/article/2013/12/03/lesclassespreparatoiresunbienprecieuxaproteger_3524479_3232.html

46 Sauf rares exceptions comme l'expulsion des philosophes en 155 avant J.-C.

47 Nicolet, Idée républicaine, p. 127 et p. 310-311.

48 Nicolet, Idée républicaine, p. 448 sq.

49 Voir J. Bleicken, “ Oberpontifex und Pontifikalkollegium: eine Studie zur römischen Sakralverfassung ", Hermes, 85, 1957 p. 347: In Rom sind staatliche und sakrale Handlungen stets eng miteinander verflochten gewesen. Die einen waren ohne die anderen undenkbar. 
pas le ius ciuile ${ }^{50}$. Certes, à l'époque de Cicéron, on rencontre des spécialistes des affaires sacrées, mais une vraie séparation des deux domaines n'était pas d'actualité. Le personnel politique de la Rome antique était aussi son personnel religieux et toute action publique se faisait après avoir interrogé les dieux ${ }^{51}$. Plus encore, la communauté romaine se conçoit aussi comme une communauté de cultes, liée à un espace particulier et aux temples qui le constituent. Le meilleur exemple du lien entre les deux se trouve sans doute ici dans le discours que Camille adresse aux Romains après la prise de Rome par les Gaulois en 390 avant J.-C. à ceux qui souhaitaient quitter une ville dévastée pour aller s'installer sur le territoire de Véies, Camille oppose le lien indissoluble qui unit la communauté romaine à ses temples et à leur emplacement physique, consacrés par les dieux ${ }^{52}$. Les dernières lignes du discours en transmettent d'ailleurs toute la substance :

Liv., 5, 54, 7 : Hic Capitolium est, ubi quondam capite humano inuento responsum est eo loco caput rerum summamque imperii fore; hic, cum augurato liberaretur Capitolium, Iuuentas Terminusque maximo gaudio patrum uestrorum moueri se non passi; hic Vestae ignes, hic ancilia caelo demissa, hic omnes propitii manentibus uobis di.

Ici se trouve le Capitole, où jadis, après la découverte d'une tête d'homme, il fut prédit que serait la tête du monde et le centre de l'Empire. Ici, lorsque les augures permirent de libérer le Capitole, la Jeunesse et Terme, à la grande joie de nos pères, ne se laissèrent pas déplacer. Ici sont les feux de Vesta, ici les boucliers envoyés du ciel, ici tous les dieux qui vous protégeront si vous restez (trad. G. Baillet).

Citons enfin le problème du rapport à l'armée. En France, depuis le 2 décembre 1851, le républicanisme et le rapport à l'armée sont problématiques. Le républicanisme ou le loyalisme de la « grande muette » ont été objets d'incessantes difficultés dans laquelle l'affaire Dreyfus n'a pas peu joué. Il en va là aussi de façon toute différente à Rome qui résout le problème de façon très simple par le lien indissoluble entre participation à l'armée et pratique politique, lien sur lequel là aussi il n'est pas utile de s'étendre ${ }^{53}$.

50 Cic., leg., 2, 47.

51 Renvoyons ici aux nombreux travaux de J. Scheid et par exemple à J. Scheid, “ Le prêtre », in A. Giardina (éd.), L'Homme romain, Paris, 1992, p. 71-106.

52 Voir ce long discours reconstruit par Liv., 5, 50-54.

53 Voir, pour mémoire, Nicolet, Métier de citoyen, p. 123 : « le Romain (tout Romain) est avant tout un guerrier, ou plutôt un soldat, c'est-à-dire moins un combattant avide d'exploits individuels qu'un citoyen discipliné incorporé dans une machine dont la redoutable efficacité provient de sa cohérence », ou p. 125-126 : “Enfin, et c'est peutêtre le plus important, le service militaire, qui finit donc par devenir facultatif pour le simple citoyen au ${ }^{\text {er }}$ siècle avant J.-C., resta obligatoire pour tous ceux qui avaient l'ambition d'aborder la carrière des honneurs ou, sous l'Empire, d'accéder aux postes 


\section{Une république relationnelle?}

Ces différents éléments peuvent conduire à détailler à présent le fonctionnement profond de la res publica libera. Partons pour cela d'un texte on ne peut plus classique : la fameuse archéologie de Rome, que l'on trouve au livre VI des Histoires de Polybe ${ }^{54}$. Sans prétendre livrer une interprétation exhaustive d'un texte qui a déjà fait l'objet de multiples commentaires, nous pouvons toutefois revenir sur son organisation et sur sa fin. Ce texte comporte trois parties principales. La première évoque le type de constitution de Rome ${ }^{55}$. On y trouve l'idée que tout État ou toute cité obéit à un fonctionnement particulier. Plus précisément encore, du point de vue des pouvoirs, ce fonctionnement se fonde sur une répartition des charges entre les différentes parties qui gouvernent. Dans le cas romain, il s'agit de trois éléments : les magistrats, le Sénat et le peuple (à travers ses assemblées, les comices). Ce point établi, Polybe passe à une description plus ou moins détaillée de ces différentes parties, en se situant au niveau des mécanismes concret d'exercice du pouvoir ${ }^{56}$. Enfin, l'analyse se poursuit par l'examen des limites et des interférences entre ces trois pouvoirs ${ }^{57}$. Ce faisant, Polybe est conduit à reprendre et à compléter certains points évoqués dans la deuxième partie du texte, le tout suivant une optique limpide : illustrer la manière dont chacune des trois parties qui gouvernent s'oppose ou collabore avec les autres, en vertu d'une distribution des compétences entre chacune d'elles.

De la sorte, cette analyse est en fait entièrement conçue en termes de rapports réciproques, de complémentarité des différents pouvoirs entre eux. La première partie du texte, où chaque élément du système romain est rapproché d'un des termes de la trichotomie classique des régimes (consul = monarchie; Sénat = aristocratie ; peuple $=$ démocratie) a souvent le plus retenu l'attention pour expliquer que Polybe présentait la constitution de Rome comme incarnant cette fameuse constitution mixte ${ }^{58}$. La partie la plus importante de ce texte est pourtant

de hauts fonctionnaires. Les classes supérieures resteront donc jusqu'à la fin soumises à des obligations militaires et tout imbues de leur esprit. Rome, en somme, même lorsqu'elle eut conquis le monde, ne s'est jamais entièrement démobilisée. »

54 Plb., 6, 11-18.

55 Plb., 6, 11, 11 à $6,11,13$.

56 Plb., 6, 12, 1 à 6, 14, 12.

57 Plb., 6, 15, 1 à $6,18$.

58 Sur cette notion, on lira encore avec profit K. von Fritz, The Theory of the Mixed Constitution in Antiquity. A Criticial Analysis of Polybius' Political Ideas, New York, 1954. Voir aussi C. CARSANA, La teoria della "Costituzione mista nell'età imperiale romana, Côme, 1990 ; E. Gabba, "L'invenzione greca della costituzione romana ", in I Greci. Storia cultura arte società, 2. Una storia greca, III. Trasformazioni, Turin, 1998, p. 857- 
bien la troisième, car on y voit que Polybe ne considère pas Rome comme un véritable exemple de constitution mixte. Rome était pour lui une aristocratie, mot à entendre dans un sens laudatif ${ }^{59}$. C'était cependant une aristocratie dont le génie politique fut moins de puiser aux trois formes pures de constitution que d'obliger en permanence, dans le champ de l'action politique, les éléments qui rappelaient ces trois formes pures à coopérer entre eux. Dans ce texte, à partir du paragraphe 6,15 , un vocabulaire bien particulier se met en place, celui de la collaboration :

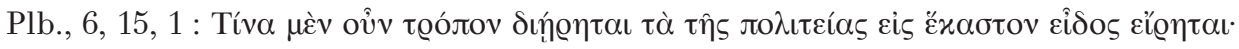

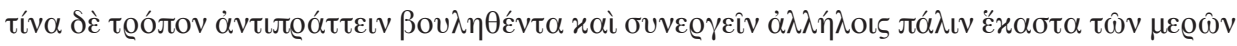

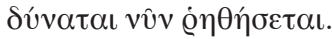

Voilà donc comment les responsabilités de l'État sont réparties entre les diverses formes de pouvoir; nous allons expliquer maintenant comment chacun de ces éléments du pouvoir peut s'il le veut s'opposer aux autres ou, au contraire, collaborer avec eux (trad. R. Weil) ${ }^{60}$.

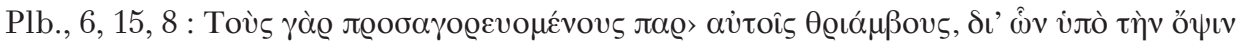
ă

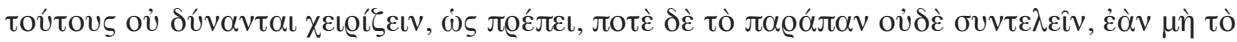

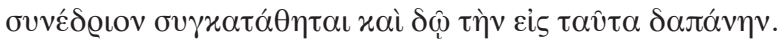

La cérémonie qu'on appelle à Rome " triomphe ", qui permet aux généraux de mettre sous les yeux de leurs concitoyens le spectacle éclatant de leurs exploits, ne peut être organisée convenablement et parfois ne peut même pas être célébrée du tout, si cette assemblée n'y consent et ne donne des fonds à cet effet (trad. R. Weil).

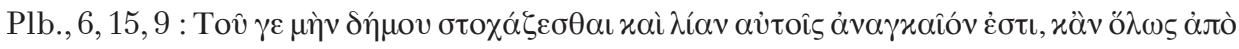

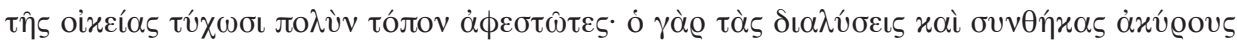

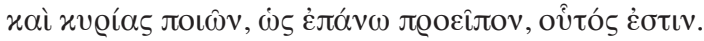

Mais les consuls doivent aussi, très nécessairement, chercher l'accord du peuple, lors même qu'une longue distance les sépare de leur patrie : celui qui sanctionne ou

867 ; F. W. Walbank, “A Greek looks at Rome: Polybius vi revisited », SCI, 17, 1998, p. 45-59 ; L. Polverini, “ Democrazia a Roma? La costituzione repubblicana secondo Polibio ", in G. Unso (éd.), Popolo e potere nel mondo antico. Concezioni Linguaggio Immagini, Pise, 2005, p. 85-96.

59 Voir Cl. Nicolet, “Polybe et la "constitution” de Rome : aristocratie et démocratie », in Id. (éd.), Demokratia et aristokratia. À propos de Caius Gracchus : mots grecs et réalités romaines, Paris, 1983, p. 18-22 et p. 31-32 ; P. Botteri et M. Raskolnikoff, “ Diodore, Caius Gracchus et la démocratie », in Nicolet (éd.), Demokratia et aristokratia, p. 8690 ; G. Zecchini, “ Polibio e la "costituzione" romana: storia di un fraintendimento », in S. Cagnazzi et alii (éd.), Scritti di Storia per Mario Pani, Bari, 2011, p. 527-528.

60 Voir aussi Plb., 6, 18, 1. 
annule les armistices et les traités, c'est lui, comme je l'ai dit tout à l'heure (trad. R. Weil) ${ }^{61}$.

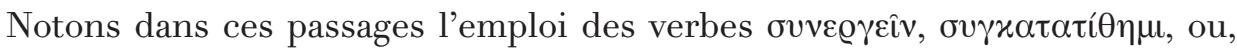

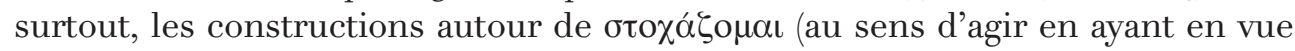
les intérêts d'une autre partie). Ce dernier exemple est des plus intéressants car il ne s'agit absolument pas d'un terme institutionnel ${ }^{62}$. C'est un terme descriptif qui cherche à rendre concrètement le dispositif décrit par Polybe : un dispositif qui pousse les acteurs à agir en tenant compte en permanence des autres pièces du jeu. D’une certaine façon, c'est une république relationnelle, une république du lien que Polybe décrit; ou, pour le dire autrement, une république qui s'intéresse moins aux aspects théoriques généraux qu'aux moyens juridiques concrets permettant de faire fonctionner l'ensemble. C'est une république du lien car le fondement du système ne repose pas sur une quelconque séparation des pouvoirs : inconnu des Romains ${ }^{63}$, ce principe est remplacé par la volonté de forcer leurs interactions, de créer un lien constant entre les trois principaux pôles qui font la vie politique romaine.

Ce lien ne se trouve pas seulement, par la vertu des interprètes modernes, dans un texte de philosophie politique grecque. Nous l'avons déjà rencontré en étudiant la notion de libertas un peu plus haut et en montrant qu'elle devait se comprendre à l'intérieur d'un réseau d'interdépendance ${ }^{64}$. Ce lien se manifeste aussi très concrètement à Rome même, dans la topographie de la ville. L'exemple du forum est à ce titre emblématique tant il s'agit d'un espace qui concentre en son sein de multiples fonctions (politique, juridique, commerciale et religieuse). Or au sein même du forum, ces différentes fonctions sont topographiquement liées, en particulier dans la zone du comitium. Le comitium, lieu primitif de réunion des assemblées du peuple, se trouve relié de façon architecturale à la curie, lieu de réunion du Sénat, et aux rostres, la tribune des orateurs. S'y trouvent aussi la graecostasis, pour la réception des ambassades, des statues et, à proximité, l'emplacement historique du tribunal du préteur. C'est également là que fut construite la première basilique (la basilica Porcia). Ce lieu offre donc une remarquable concentration de l'incarnation physique des différentes fonctions politiques de Rome, concentration qui semble bien avoir survécu aux multiples transformations de cet espace. Nous touchons ici à un problème

61 Voir aussi Plb., 6, 16, 1 ; Plb., 6, 16, 5 ou encore Plb., 6, 17, 1.

62 H. J. Mason, Greek Terms for Roman Institutions, Toronto, 1974 l'ignore, par exemple.

63 Schulz, Prinzipien, p. 122. Voir aussi Hurlet, “Démocratie à Rome? Quelle démocratie? », p. 43. La notion de participation était d'ailleurs un concept clef de Fergus Millar.

64 Cf. supra p. 146-147. 
complexe d'archéologie, qui est celui de l'histoire du comitium et de son possible changement de forme. Les travaux de C. M. Amici fournissent toutefois de bons indices archéologiques pour abandonner l'idée du passage d'un comitium carré à un comitium rond, d'une part, et, d'autre part, pour souligner que les différentes curies furent toutes construites en réalité sur la même zone, suivant la même orientation et toujours en relation fonctionnelle avec la zone du comitium, située légèrement en contrebas ${ }^{65}$. Le caractère lié de ces différents espaces aurait de la sorte perduré jusqu'à la fin de la République. Même lorsque, durant le $\mathrm{I}^{\mathrm{er}}$ siècle avant J.-C., on assiste à un glissement des activités vers le Sud-Est du forum, ce glissement se fit par groupe : le tribunal du préteur, mais aussi, ensuite, certaines opérations de vote au temple de Castor et Pollux (qui servait parfois de lieu de réunion du Sénat), tandis qu'on utilisa les rostres du divin Jules comme tribune. À une époque où les institutions de la République s'évanouissaient, une petite zone similaire, toute proportion gardée, à ce qu'avait été le comitium, se récréait alors dans cette partie Sud-Est du forum, avant que les modifications césaroaugustéenne du forum ne transforment définitivement cet espace. D'une certaine façon, si, dans nos républiques modernes, l'impératif de séparation des pouvoirs va jusqu'à s'incarner dans une séparation physique des acteurs du pouvoir, il en va de manière totalement inverse à Rome jusqu'à la mise sur pied du Principat.

On pourrait bien sûr se demander (cela a été fait de nombreuses fois) dans quelle mesure la description de Polybe est opératoire, surtout à partir du moment où il ne souffle mot de la structure censitaire de la société romaine. S'il n'en souffle mot, c'est cependant précisément parce qu'il veut mettre l'accent sur ces questions relationnelles, qui lui semblent tout aussi vitales et moins visibles qu'une structure censitaire qui ne pouvait échapper à un observateur attentif de la vie politique romaine. D'après Cl. Nicolet, les républicains français s'interrogeaient essentiellement sur le problème de la participation au pouvoir ${ }^{66}$. Inversement, chez les Anglais ou chez les libéraux tout tourne autour d'une conception de la limitation des pouvoirs. Je voudrais suggérer que, à Rome, le problème est plutôt pensé en termes d'interrelations des différents acteurs du pouvoir : tout est fait pour les pousser à la collaboration. Pensons à la collégialité, à l'annualité, à la prouocatio, ou à l'intercessio. Le système, lentement élaboré durant les deux

65 Sur ces questions complexes de modification du comitium, voir F. Coarelli, "Comitium », dans LTUR, 1, 1993, p. 309-314; P. CARAFa, Il Comizio di Roma dalle origini all'età di Augusto, Rome, 1998 ; M. Huмм, « Le comitium du forum romain et la réforme des tribus d'Appius Claudius Caecus ", MEFRA, 111/2, 1999, p. 625-694 ; С. М. Амгсг, “Evoluzione architettonica del comizio a Roma », RPAA, 77, 2004-2005, p. 351-379; P. Carafa, “Il Volcanal e il comizio », WAC, 2, 2005, p. 135-149 et M. Humm, Appius Claudius Caecus. La République accomplie, Rome, 2005, p. 601-638.

66 Nicolet, Idée républicaine, p. 357 
premiers siècles de la République, tourne entièrement autour de ce problème qu'efface en un sens la reconstruction de Th. Mommsen puisque, comme l'avait bien remarqué Cl. Nicolet, Th. Mommsen et ses épigones concevaient l'histoire républicaine de Rome comme un champ d'application de l'implicite théorie des pouvoirs qui sous-tend leur œuvre, masquant d'une certaine manière la spécificité de la construction politique romaine ${ }^{67}$.

\section{"République " : un dispositif de neutralisation du politique ?}

Venons-en alors à une proposition plus générale visant à montrer en quoi République romaine et république contemporaine peuvent encore trouver un intéressant point de rapprochement, à partir des idées de J. Rancière. Pour le dire d'emblée, on peut se demander si l'architecture institutionnelle de la res publica classique, tout comme celles de nos républiques respectives (en particulier celle de la $\mathrm{V}^{\mathrm{e}}$ République), n'ont pas comme ligne d'horizon la même finalité : l'effacement du politique. Il faut, pour le comprendre, revenir quelques instants sur les idées de J. Rancière et, en particulier sur la différence très nette qu'il établit entre police et politique. Pour J. Rancière, la police désigne «l'ensemble des processus par lesquels s'opèrent l'agrégation et le consentement des collectivités, l'organisation des pouvoirs, la distribution des places et fonctions et les systèmes de légitimation de cette distribution ${ }^{68}$ ». Il donne donc un sens large à ce terme, qui recouvre ce que l'on appelle généralement police, mais aussi tous les processus de gouvernement. Inversement, il donne au terme de " politique » un sens beaucoup plus restreint, ce qui le conduit à écrire qu'il n’y a, en réalité, que rarement de la politique au sens fort ${ }^{69}$. La politique devient alors précisément le moment où la police, comprise comme ce partage et cette organisation du sensible, est remise en question. La politique est le moment d'interrogation du partage du commun dans le but d'instaurer véritablement un commun, une scène commune, qui fasse une part à ce que J. Rancière dénomme les sans-parts. Inversement, la philosophie dite politique, correspond pour lui à l'ensemble des opérations de pensée par lesquelles cette politique au sens fort doit être liquidée $\mathrm{e}^{70}$.

J. Rancière reconnaît trois grands courants dans la philosophie politique, incarnés chacun par une figure tutélaire: l'archi-politique (Platon), la parapolitique (Aristote) et la méta-politique (Marx). Chacune de ces trois formes de

67 Cl. Nicolet, “ Prosopographie et histoire sociale : Rome et l'Italie à l'époque républicaine », Annales ESC, 25/5, 1970, p. 1214 n. 4.

68 J. Rancière, La Mésentente. Politique et philosophie, Paris, 1995, p. 51.

69 Rancière, Mésentente, p. 37.

70 Rancière, Mésentente, p. 15. 
la philosophie politique serait une manière d'évacuer la politique du champ de la cité. L'archi-politique platonicienne se réalise par la suppression totale de l'espace commun et de la politique au profit d'une communauté considérée comme un ensemble clos, organisée sur une division intelligente des tâches (pensons à la fable des quatre travailleurs) et chapeautée par le projet épistémologique et éducatif du philosophe roi. Si J. Rancière peut écrire de l'archi-politique

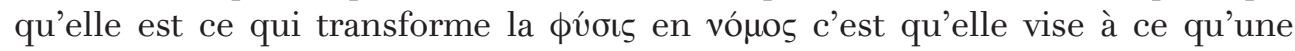
répartition intelligente selon la nature soit au cœur du projet. La para-politique aristotélicienne se détache, elle, de l'ambition de Platon qui évacue en fait quasi complètement la politique. Son but est bien sûr de contenir là aussi les excès de la démocratie (de la politique dirait J. Rancière) mais cela passe cette fois par la mise sur pied d'institutions politiques adéquates qui encadrent et réduisent l'activité proprement politique, d'où le fait que J. Rancière puisse indiquer que la para-politique tend à identifier activité politique et ordre policier. Elle veut les faire coïncider. C'est cette tradition para-politique qui fut réactivée par les théoriciens modernes du contrat, dont on sait l'influence qu'ils eurent sur l'idée républicaine en Europe et singulièrement en France, à travers Rousseau. Reste la méta-politique, le projet spécifiquement marxiste, qui nous intéresse moins ici.

Ce détour permet de comprendre l'idée que je souhaiterais suggérer, surtout après ce qui a été avancé de Rome comme république relationnelle. Le magnifique dispositif institutionnel républicain ne serait-il pas l'incarnation même du projet para-politique? Et puisque ce projet fut réitéré par les théoriciens modernes du contrat, ne peut-on rapprocher ces deux tentatives pour y voir à chaque fois des régimes d'évacuation du politique compris dans sa forme radicale, celle de la mésentente, celle du conflit pour donner une part aux sans-part?

Il est alors intéressant de revenir ici sur la périodisation de l'histoire romaine républicaine. Tout notre propos a en réalité essentiellement concerné les institutions républicaines classiques, telles qu'elles se mettent définitivement en place à la fin du rv siècle avant J.-C. et qui perdurèrent jusqu'à la chute de ce régime, même à travers les vicissitudes du $\mathrm{I}^{\text {er }}$ siècle avant J.-C. Or, à la fin du $\mathrm{Iv}^{\mathrm{e}}$ siècle avant J.-C., la République avait déjà deux siècles d'existence, mais ces deux siècles sont un peu différents. Ce sont ceux du fameux conflit des ordres. L'histoire de ces deux siècles peut s'analyser notamment à travers le prisme des tribuns de la plèbe qui en furent des acteurs majeurs. Ces tribuns furent créés suite à la sécession de la plèbe en 494 avant J.-C., sécession qui fut un événement majeur ainsi qu'un acte politique au sens le plus fort, y compris au sens de J. Rancière. Par leur action, notamment par leurs plébiscites, les tribuns de la plèbe jouèrent un rôle tout à fait important dans l'établissement des institutions républicaines classiques. Ils participèrent de l'encadrement de l'imperium consulaire, ils créèrent le principe de l'assemblée tribute, ils furent à l'origine du Sénat républicain classique, ils promurent le rôle du peuple en matière 
criminelle, ils attaquèrent et entamèrent sérieusement la prétention patricienne au monopole religieux. Rappelons également le rôle des tribuns dans la mise en place du principe de collégialité des magistratures, le rôle du plébiscite de 367 avant J.-C. dans la création de la nobilitas, ou encore le rôle important du plébiscite ogulnien dans l'accès des plébéiens aux fonctions religieuses. Enfin, de façon plus souterraine, les tribuns jouèrent un rôle évident dans l'élaboration de la notion classique de populus, à la fois par l'invention d'une assemblée tribute et par la promotion du plébiscite comme source normative. De toute évidence, les tribuns de la plèbe ne créèrent pas, à eux seuls, en fonction d'un programme préétabli, les institutions républicaines ; ils le firent aussi parce qu'ils défendaient l'intérêt bien compris d'un certain groupe social. Ils n'en demeurent pas moins des acteurs majeurs des processus qui finirent par donner naissance à la République romaine classique $^{71}$.

L'établissement de cette république classique ne serait-il cependant pas l'aboutissement de ce processus d'évacuation de la politique, de sa neutralisation par un ingénieux système d'interrelations, lequel ne fut brisé que par le resurgissement de la question politique, durant le II $^{\mathrm{e}}$ siècle avant J.-C., avec l'action des Gracques? Sur le fond, cette République dite classique ne se rapproche-t-elle pas des objectifs concrets actuels de nos démocraties consensuelles ? Toute une série de travaux historiques, particulièrement ceux de l'école allemande (en particulier les élèves de Chr. Meier) ont cherché à décrire la république romaine comme fonctionnant de manière à créer du consensus ${ }^{72}$. La présentation de J. Rancière permet peut-être d'aller encore au-delà de cette présentation pour en dévoiler les soubassements : l'évacuation du politique. Le lecteur nous autorisera alors une forme de proposition paradoxale, à savoir que si Rome a jamais été un régime faisant place au politique, ce fut peut-être uniquement au plus fort de ses périodes de conflit, et notamment durant les $v^{\mathrm{e}}$ et $\mathrm{rv}^{\mathrm{e}}$ siècles avant J.-C.

Faisons alors un ultime pas dans cette direction en pensant ici à la façon dont Cl. Lefort, de façon analogue à J. Rancière, définit non plus la politique cette fois, mais la démocratie. La démocratie correspond pour lui au système qui institutionnalise le conflit, qui en permet l'expression et le considère même légitime, précisément parce que ce qui caractérise ce régime c'est que le lieu du pouvoir est vide : il n’est pas incarné, mais est successivement occupé par

71 Sur tous ces aspects, voir Lanfranchi, Tribuns de la plèbe et M. Humbert, « L'inspiration démocratique de l'insurrection de la plèbe (494) : les institutions plébéiennes, les XII Tables et les plébiscites », $R D, 93 / 3,2015$, p. 393-443.

72 Voir K.J. Holkeskamp, Reconstruire une République. La “culture politique » de la Rome antique et la recherche des dernières décennies, Nantes, 2008 pour appréhender ce courant de recherche. 
des groupes différents qui s'opposent pour y accéder. La véritable politique, la véritable démocratie c'est donc celle où la division est reconnue et trouve place dans l'espace public ${ }^{73}$. À Rome, c'est bien durant les deux premiers siècles de la république que cela se produisit. Bien que le trait soit quelque peu forcé ici, un tel renversement de perspective me paraît porteur d'éléments féconds pour la compréhension du fonctionnement de la République romaine et pour sa comparaison avec les républiques modernes. La République romaine était bien, d'une certaine manière, républicaine et présente de réelles convergences de fond avec des républiques très différentes par ailleurs. C'était un régime caractérisé par une organisation très fine des relations de pouvoir, issue de deux siècles d'intenses luttes politiques, et visant à assurer in fine la domination de la nobilitas. C'était un régime de neutralisation du politique, même si le sens exact de ce que les Romains nommaient politique reste encore à définir.

\section{Thibaud Lanfranchi}

Maître de Conférences d'Histoire romaine Université Toulouse - Jean Jaurès (UT2J)

Maison de la Recherche

PLH-ERASME (EA 4601)

lanfranc@univ-tlse2.fr

73 Voir, par exemple, Cl. Lefort, L'Invention démocratique. Les limites de la domination totalitaire, Paris, 1981. 